Supporting Information

\title{
Highly Monodisperse Cu-Sn Alloy Nanoplates for Efficient Nitrophenol Reduction Reaction via Promotion Effect of $\mathrm{Sn}$
}

Zhenxing Li, ${ }^{*}{ }^{\dagger}, \S$ Miao He, ${ }^{\dagger}, \S$ Yangyang Wen, ${ }^{\dagger}$ Xin Zhang, ${ }^{\dagger}$ Mingliang Hu,,$^{\dagger}$ Rui Li,$^{\dagger}$ Jiahao Liu,,$^{\dagger}$ Junmei Chu,,$^{\dagger}$ Zhengzheng Ma, Xiaofei Xing,,$^{\dagger}$ Chengcheng Yu, ${ }^{\dagger}$ Zhiting Wei, ${ }^{\dagger}$ and Yongle Li $i^{*,+}$

†State Key Laboratory of Heavy Oil Processing, College of New Energy and Materials, Beijing Key Laboratory of Biogas Upgrading Utilization, China University of Petroleum (Beijing), Beijing 102249, China

*Department of Physics, International Center for Quantum and Molecular Structures, and Shanghai Key Laboratory of High Temperature Superconductors, Shanghai University, Shanghai 200444, China 


\section{EXPERIMENTAL SECTION}

Preparation of $\mathbf{C u}-\mathrm{Sn}$ nanoplates and $\mathrm{Cu}$ nanoparticles. In this synthesis, $0.4 \mathrm{mmol} \mathrm{SnCl}_{2}, 0.8 \mathrm{mmol}$ $\mathrm{CuCl}_{2}$ and $15 \mathrm{~mL}$ of oleylamine were added into $100 \mathrm{~mL}$ three-necked flask, and heated the mixture with stirring to $140{ }^{\circ} \mathrm{C}$ for 45 minutes under the vacuum condition, after that, raised the temperature to $230{ }^{\circ} \mathrm{C}$, kept this temperature for 3 hours under the Ar condition, and cooled the mixture immediately to the room temperature by ice water. The $\mathrm{Cu}$ nanoparticles was prepared by adding $0.1 \mathrm{~g}$ Copper acetylacetonate, $0.06 \mathrm{~g}$ ascorbic acid and $5 \mathrm{~mL}$ of oleylamine, and heating the mixture at $180{ }^{\circ} \mathrm{C}$ in an oil bath. Both the nanoparticles and nanoplates were dispersed in $10 \mathrm{ml}$ of chloroform, sonicated for 30 min, and then added to a stirred chloroform solution containing $0.1 \mathrm{~g}$ polyvinyl pyrrolidone, stirred overnight, and washed with n-hexane to remove chloroform.

Catalytic reduction of 4-nitrophenol. In a typical experiment, $0.2 \mathrm{ml}$ of $1 \mathrm{mM}$ 4-NP solution was mixed with $1.5 \mathrm{ml}$ of deionized water, and then $0.5 \mathrm{ml}$ of a freshly made $0.5 \mathrm{M} \mathrm{NaBH}_{4}$ solution was added. Afterwards, $0.2 \mathrm{ml}$ of catalyst suspension $(0.556 \mathrm{mg} / \mathrm{L}$, carried out by ICP-OES) was added and the mixed solution was placed in a UV-vis spectrophotometer and scanned in the range of 250-500 $\mathrm{nm}$

Catalytic reduction of other organic dyes. In this experiment, $2.5 \mathrm{ml}$ of $0.05 \mathrm{mM} \mathrm{MB}, \mathrm{MO}, \mathrm{RhB}$ or industrial wastewater solution were mixed with $0.5 \mathrm{ml}$ of $0.5 \mathrm{M}$ fresh $\mathrm{NaBH}_{4}$ solution. Subsequently, $0.2 \mathrm{ml}$ of catalyst suspension $(0.556 \mathrm{mg} / \mathrm{L}$, carried out by ICP-OES) was added. The mixed solutions were placed in the UV-vis spectrophotometer and scanned as described previously.

Characterization. The transmission electron microscopy (TEM) was per-formed on a JEM 2100 LaB6 at an accelerating voltage of $200 \mathrm{kV}$. The energy-dispersive X-ray spectroscopy (EDS) was recorded on a Hitachi SU8010 scanning electron microscopy under a working voltage of $200 \mathrm{kV}$. The small-angle and wide-angle X-ray diffraction (XRD) patterns were measured on a Burker D8-advance X-ray power diffractometer operated at $40 \mathrm{kV}$ and current of $40 \mathrm{~mA}$ with $\mathrm{Cu}-\mathrm{K} \alpha$ radiation $(\lambda=1.5406 \AA)$. The actual $\mathrm{Cu}$ element content in the catalysts was determined using inductively coupled plasma-optical emission 
spectroscopy (ICP-OES) with a PerkinElmer emission spectrometer. Scanning electron microscopy (SEM) was carried out using a FEI Quanta 200F. The X-ray photoelectron spectrometers (XPS) were performed at an ion-pumped chamber (evacuated to $2 \times 10^{-9}$ Torr of an Escalad5 spectrometer, employing Mg KR radiation (BE) $1253.6 \mathrm{eV}$ ). The ultraviolet-visible (UV-vis) absorbance spectra were obtained using a UV-9000 double beam spectrophotometer with a wavelength range of 200-1000 nm. Fourier transform infrared (FT-IR) spectra were recorded on a PerkinElmer Spectrum One FT-IR spectrophotometer using the potassium bromide pellet technique. Mass spectrum was detected at the gas chromatography-mass spectrometry (GC-MS) with an Agilent 7890B gas chromatograph. 


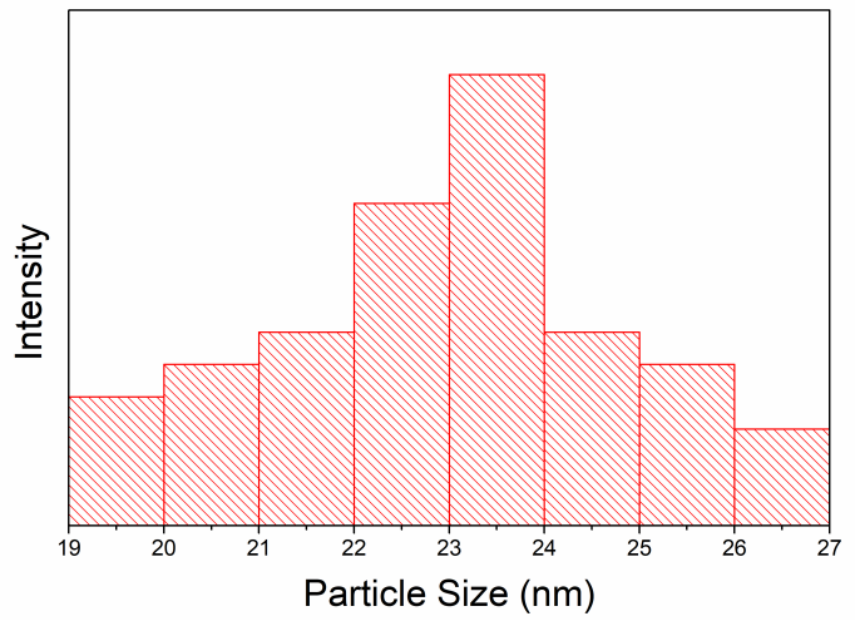

Figure S1. The images of particle size distribution of Figure 1a. 
(a)

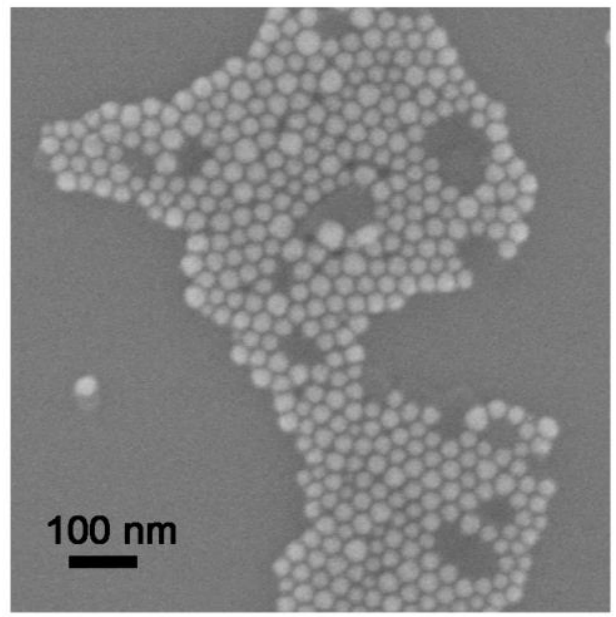

(b)

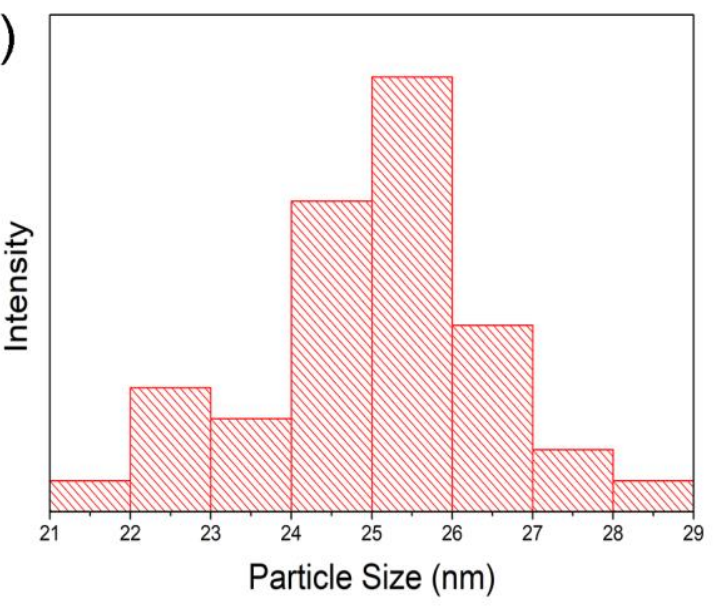

Figure S2. Images of (a) SEM and (b) particle size distribution of the Cu nanoparticles. 


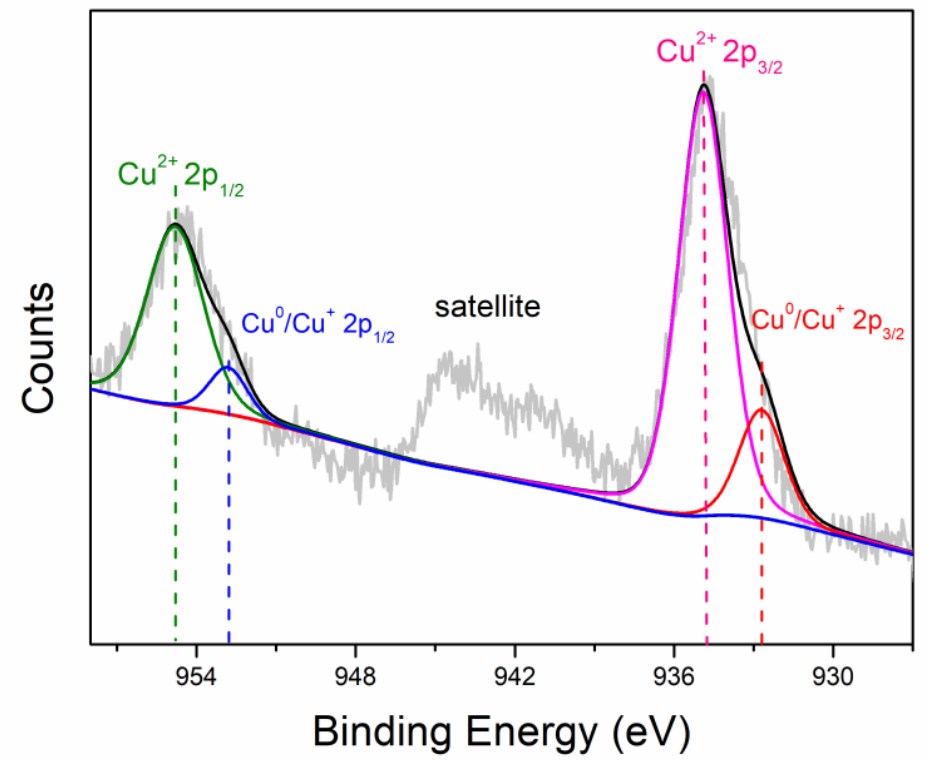

Figure S3. $\mathrm{Cu} 2 \mathrm{p}$ XPS spectrum of the $\mathrm{Cu}$ nanoparticles. 


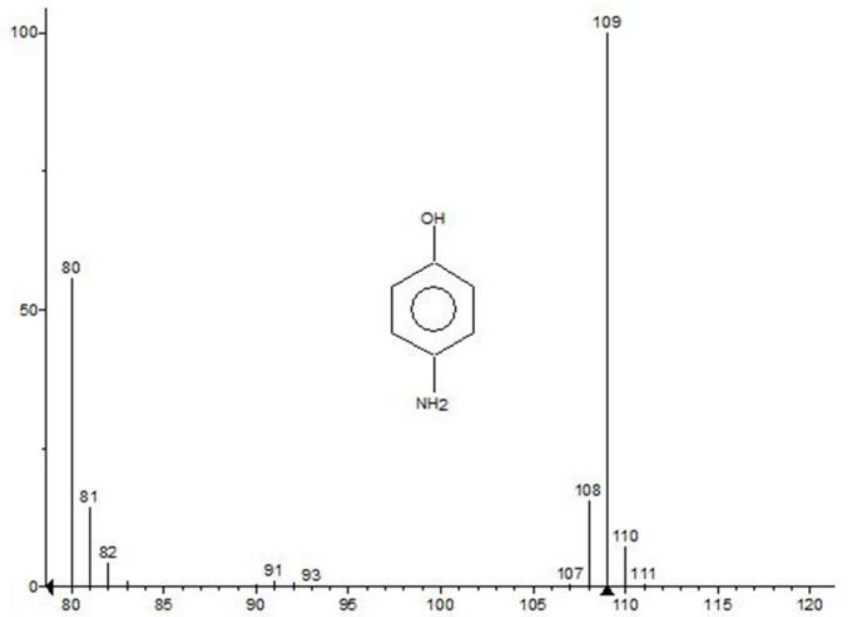

Figure S4. The mass spectrum of 4-AP. 

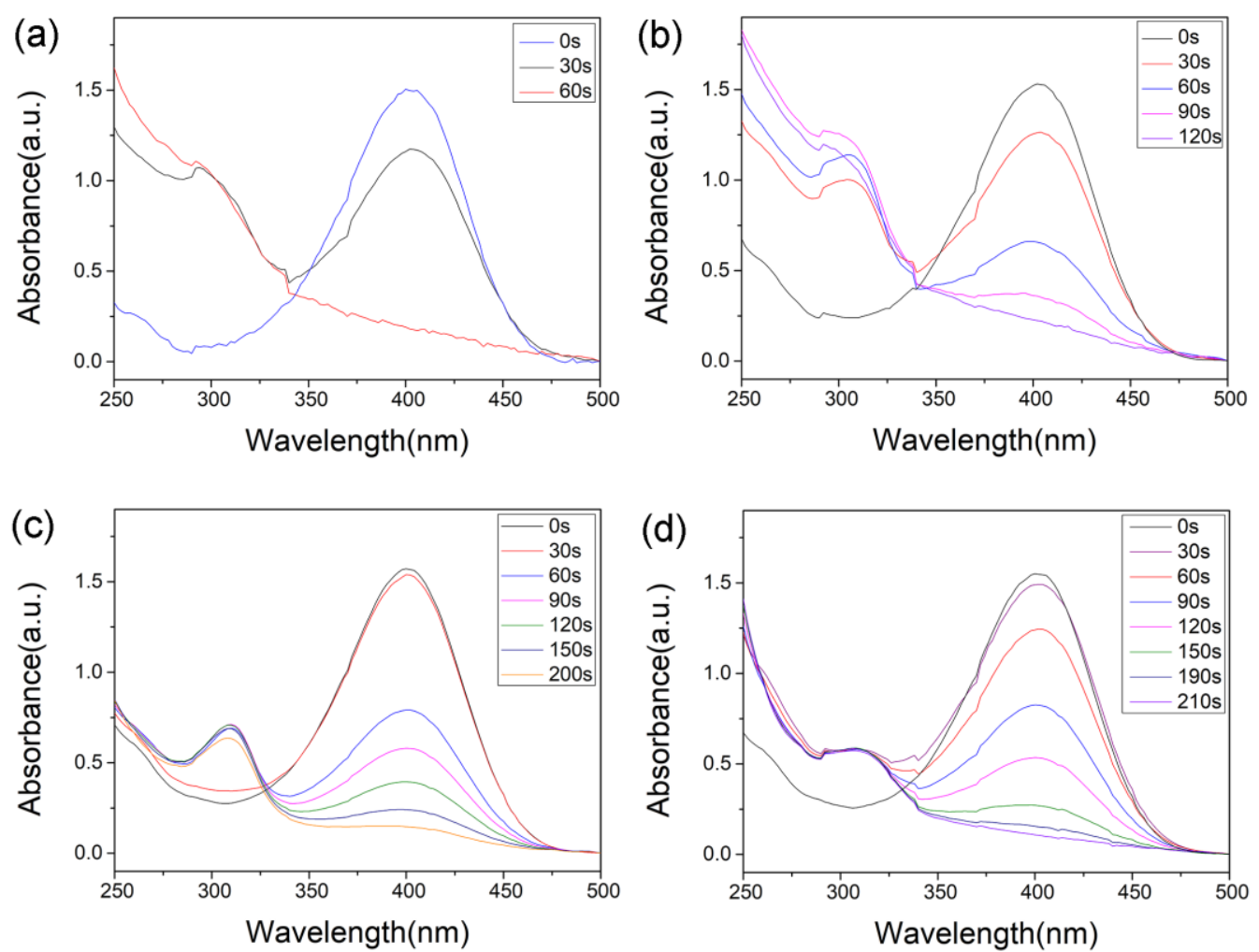

Figure S5. The UV-vis adsorption spectra for the reduction reaction of 4-NP with the solution which were (a) $\mathrm{pH}=2$, (b) $\mathrm{pH}=4$, (c) $\mathrm{pH}=11$, (d) $\mathrm{pH}=13$. 

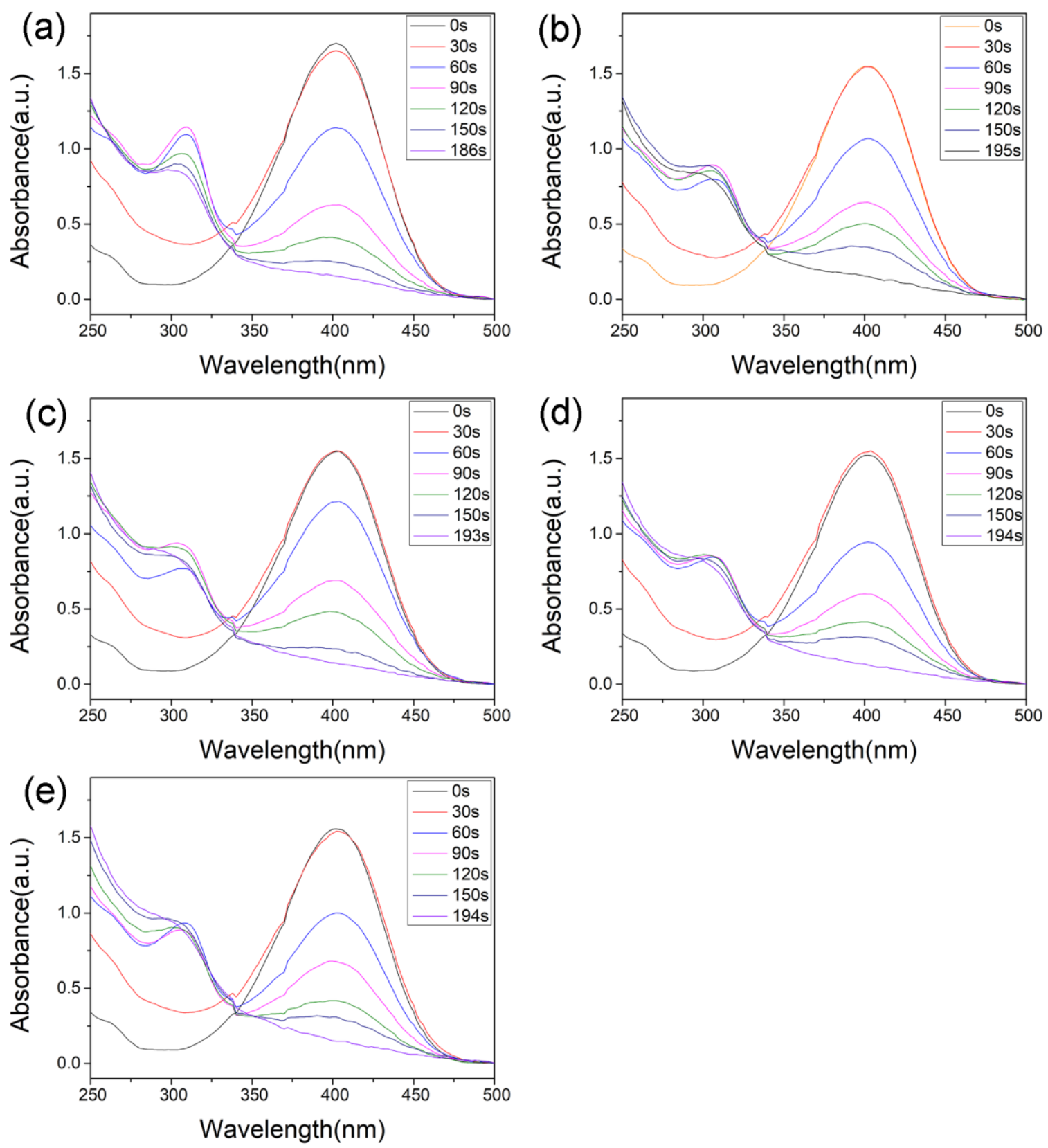

Figure S6. The UV-vis adsorption spectra for the reduction reaction of 4-NP on $\mathrm{Cu}-\mathrm{Sn}$ nanoplates with different $\mathrm{NaCl}$ concentration which were (a) $0.2 \mathrm{M}$, (b) $0.4 \mathrm{M}$, (c) $0.6 \mathrm{M}$, (d) $0.8 \mathrm{M}$, (e) $1.0 \mathrm{M}$. 

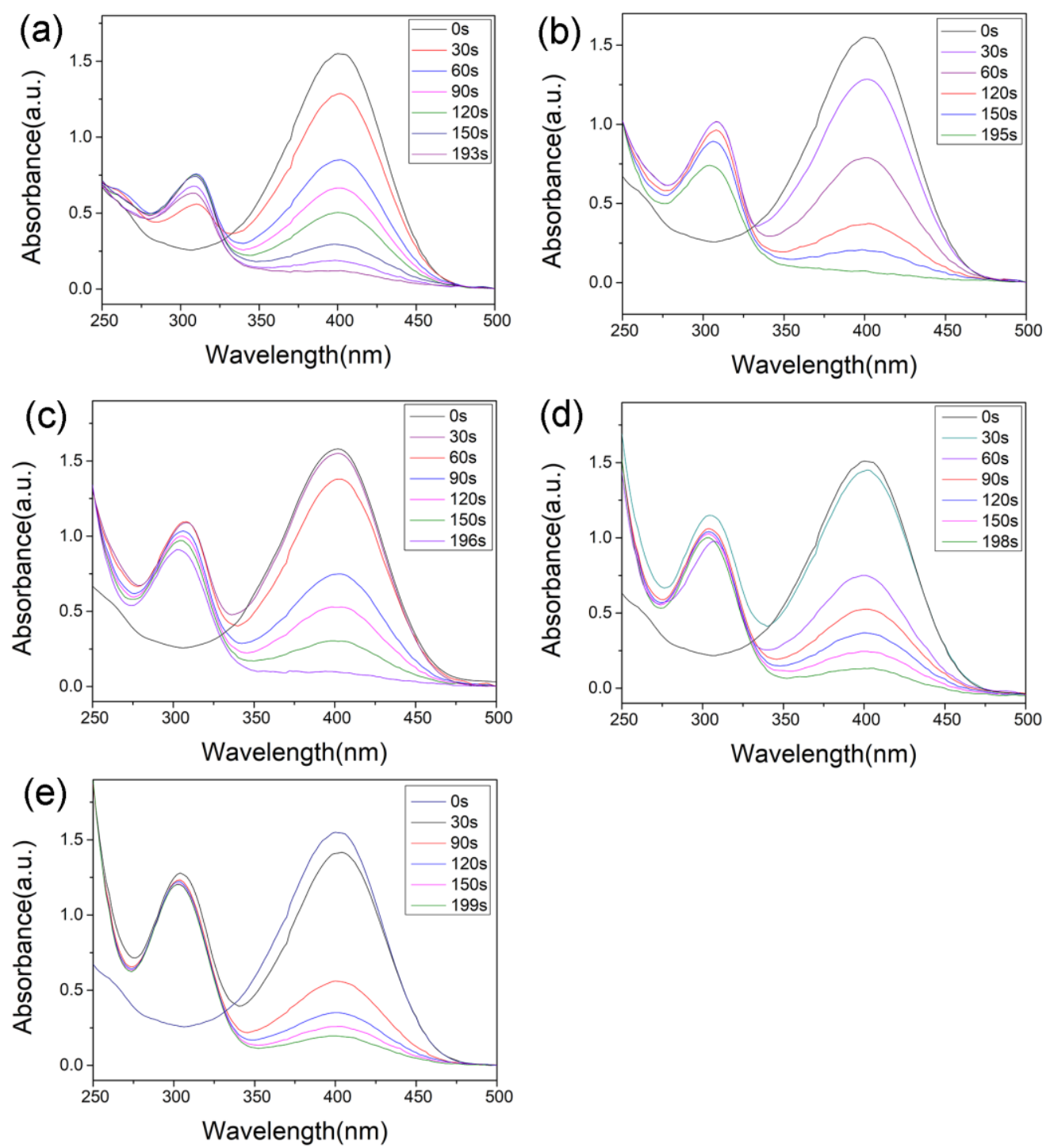

Figure S7. The UV-vis adsorption spectra for reduction reaction of 4-NP on $\mathrm{Cu}-\mathrm{Sn}$ nanoplates after (a) the first, (b)the second, (c) the third, (d) the fourth and (e) the fifth cycle used 
(a)

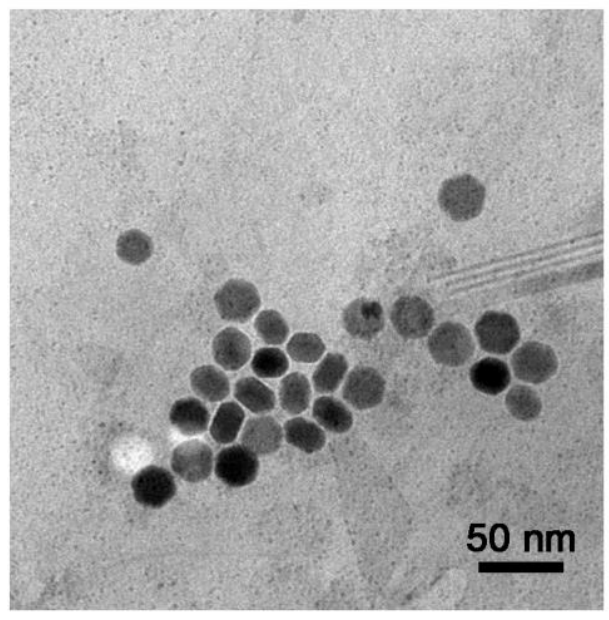

(b)

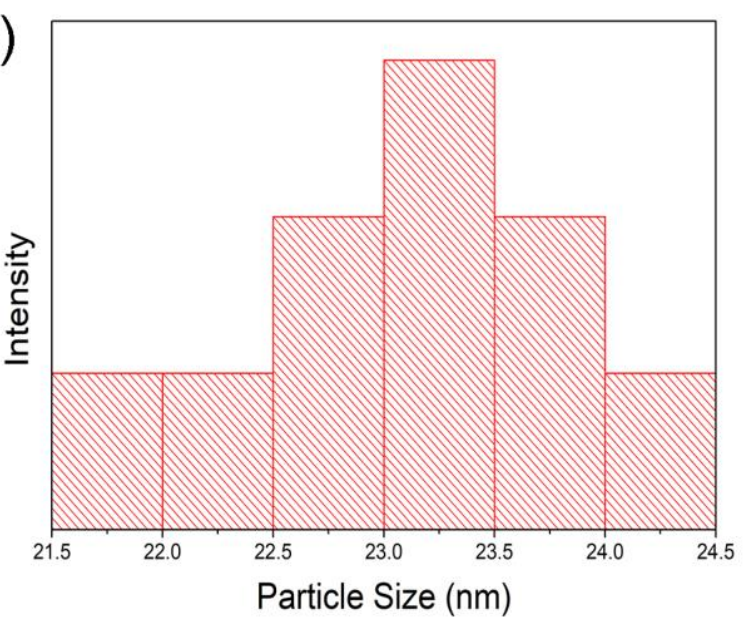

Figure S8. Images of (a) TEM and (b) particle size distribution of the $\mathrm{Cu}-\mathrm{Sn}$ nanoplates after five cycling catalytic reaction. 

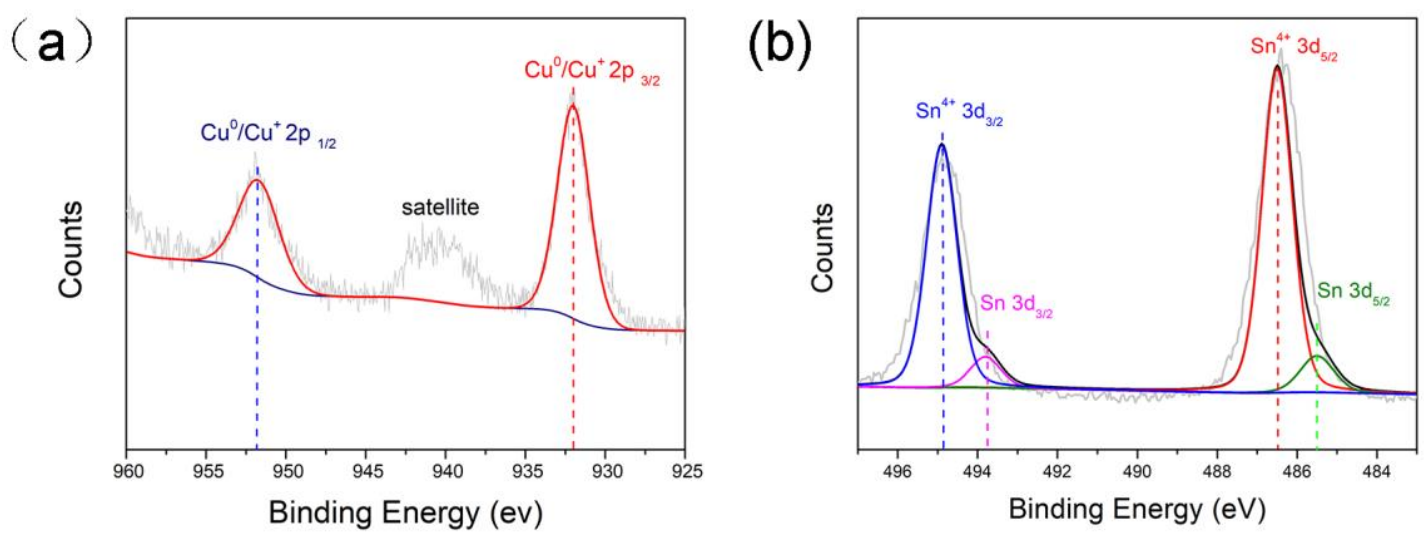

Figure S9. (a) $\mathrm{Cu} 2 \mathrm{p}$ XPS spectrum of the Cu-Sn nanoplates after the reaction. (b) Sn 3d XPS spectrum of the $\mathrm{Cu}-\mathrm{Sn}$ nanoplates after the reaction. 


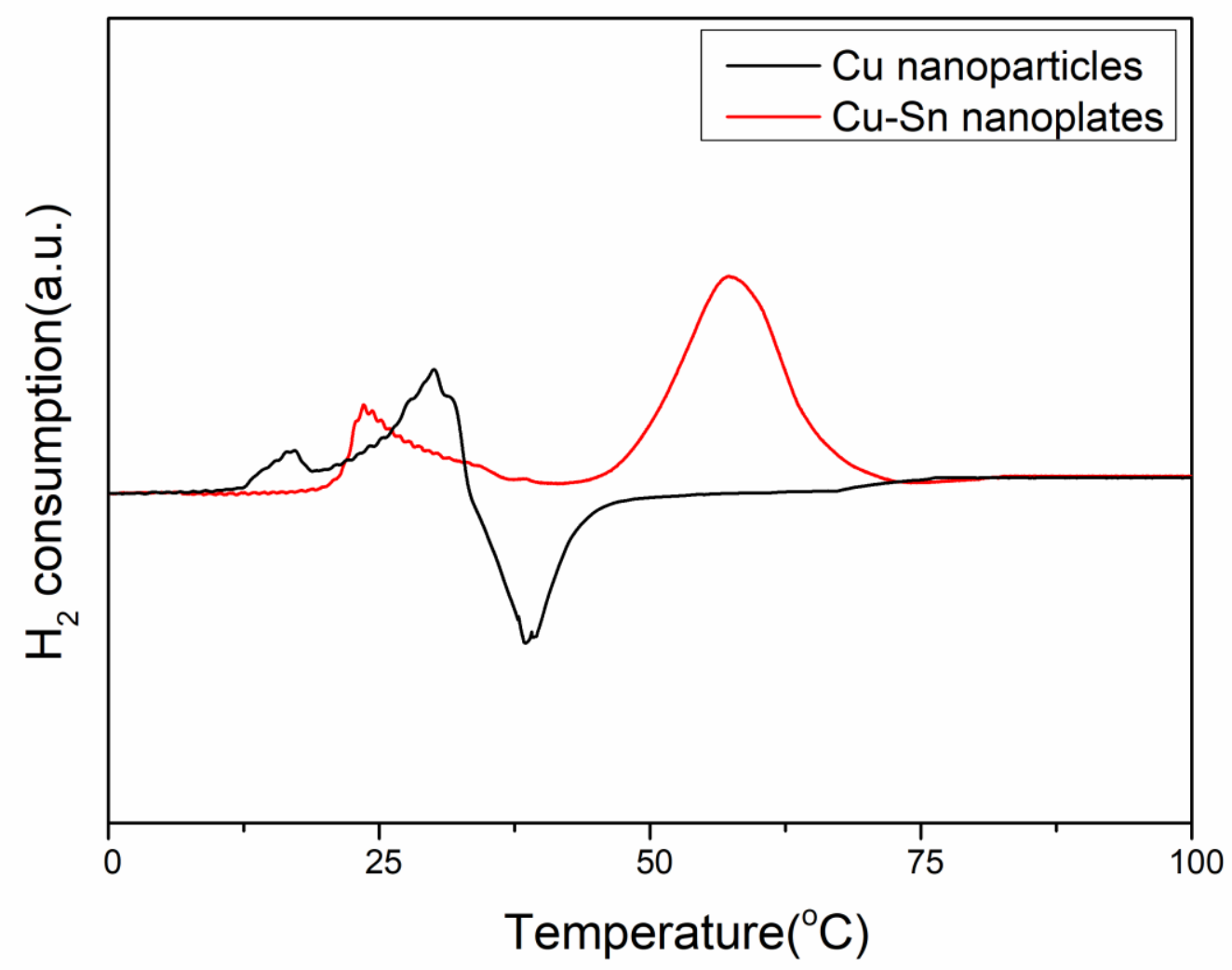

Figure S10. $\mathrm{H}_{2}$-TPR profiles of the $\mathrm{Cu}-\mathrm{Sn}$ nanoplates and $\mathrm{Cu}$ nanoparticles catalysts with the heating rate of $10^{\circ} \mathrm{C} / \mathrm{min}, \mathrm{m}_{\mathrm{Cu}}=0.5 \mathrm{~g}, \mathrm{~m}_{\mathrm{Cu}-\mathrm{Sn}}=0.2 \mathrm{~g}$. 
Table S1. The catalytic activity of various catalysts for 4-NP reduction.

\begin{tabular}{ccccccc}
\hline Catalysts & $\begin{array}{c}\text { Concentration } \\
\text { of 4-NP } \\
\left(\mathrm{mmolL}^{-1}\right)\end{array}$ & $\begin{array}{c}\text { Catalyst } \\
\text { usage }\end{array}$ & $\begin{array}{c}\mathrm{NaBH}_{4} \\
\text { usage }\end{array}$ & $\begin{array}{c}\text { Reaction } \\
\text { Time }\end{array}$ & $k_{\mathrm{a}}$ & Ref. \\
$\left(\times 10^{-3} \mathrm{~s}^{-1}\right)$ & \\
\hline $\mathrm{Cu}-\mathrm{Sn} \mathrm{NPs}$ & 1 & 0.11 & 9.46 & 190 & 17.6 & This work \\
$\mathrm{Cu} \mathrm{NPs}$ & 1 & 0.11 & 9.46 & 540 & 5.3 & This work \\
$\mathrm{Cu} / \mathrm{BNO}$ & 5 & 5 & 4.73 & 300 & 11.5 & 47 \\
$\mathrm{CuO}$ & 0.2 & 0.01 & 11.35 & 1200 & 1.95 & 48 \\
$\mathrm{Ag} @ \mathrm{Pt} / \mathrm{saprolite}$ & 0.2 & 1000 & 15.13 & 900 & 5 & 49 \\
$\mathrm{Ag} / \mathrm{KCC}-1$ & 0.12 & 0.2 & 9.46 & 510 & 10 & 50 \\
$\mathrm{Ag}-\mathrm{Au}$ & 5 & 0.1 & 0.076 & 360 & 3.47 & 51 \\
$\mathrm{Cu} 2 \mathrm{O}-\mathrm{Cu}-\mathrm{CuO}$ & 0.3 & 1 & 0.2 & 180 & 15.6 & 34 \\
$\mathrm{Pt}-\mathrm{Au}-\mathrm{PDA} / \mathrm{RGO}$ & 0.1 & 0.06 & 1.13 & 720 & 3.43 & 52 \\
$\mathrm{CuO}-\mathrm{S}-\mathrm{N}(\mathrm{OH})_{2}$ & 2.5 & 0.1 & 7.57 & 480 & 4.17 & 53 \\
\hline
\end{tabular}


Table S2. The TOF values of various catalysts for 4-NP reduction.

\begin{tabular}{ccc}
\hline Catalysts & TOF & Ref. \\
& $\left(\mathrm{min}^{-1}\right)$ & \\
\hline $\mathrm{Cu}-\mathrm{Sn} \mathrm{NPs}$ & 37.13 & This work \\
$\mathrm{Fe}_{3} \mathrm{O}_{4}-\mathrm{Ni}$ & 0.024 & 1 \\
$\mathrm{Cu} / \mathrm{Co} @ \mathrm{NCF}-15$ & 7.26 & 2 \\
$\mathrm{SiNWAs}-\mathrm{Cu}$ & 3.67 & 3 \\
$\mathrm{Pt}_{3} \mathrm{Au}_{1}-\mathrm{PDA} / \mathrm{RGO}$ & 3.33 & 4 \\
$\mathrm{Ru} / \mathrm{MgLaO}$ & 0.022 & 5 \\
$\mathrm{Cu} / \mathrm{BNO}$ & 0.45 & 47 \\
\hline
\end{tabular}


Table S3. The quality index of industrial wastewater

\begin{tabular}{cc}
\hline quality index & industrial wastewater \\
\hline $\mathrm{pH}$ & 7.28 \\
$\mathrm{COD}(\mathrm{mg} / \mathrm{L})$ & 27695 \\
$\mathrm{BOD}(\mathrm{mg} / \mathrm{L})$ & 2170 \\
Total nitrogen concentration $(\mathrm{mg} / \mathrm{L})$ & 964.5 \\
Ammonia nitrogen concentration $(\mathrm{mg} / \mathrm{L})$ & 527.5 \\
Nitrate nitrogen concentration $(\mathrm{mg} / \mathrm{L})$ & 87.5 \\
Salinity $(\mathrm{mg} / \mathrm{L})$ & 10250 \\
\hline
\end{tabular}




\section{Computational details}

All calculations were performed with Gaussian 16 software package, version A.03. ${ }^{6}$ The density functional theory (DFT) method with the $\omega$ B97X-D functional ${ }^{7}$ was utilized to optimize the stationary points without symmetry and geometric constraints, in conjuction with the standard $6-31 \mathrm{G}(\mathrm{d}, \mathrm{p})$ basis set. Harmonic vibration frequency calculations at the same level were performed to confirm the nature

of the stationary points. Both the transition state structures are validated by Intrinsic reaction coordinates (IRC) scan. 
Table S4. Energies optimized for Figure 9a at $\omega$ B97X-D/6-31G (d,p) level (300K,1 atm)

\begin{tabular}{cccc}
\hline & $\mathrm{A}$ & $\mathrm{TS}_{\mathrm{A}-\mathrm{B}}$ & $\mathrm{B}$ \\
\hline $\mathrm{E}$ (Hartree) & -906.48231 & -906.43816 & -906.48852 \\
$\mathrm{G}$ (Hartree) & -906.41351 & -906.37366 & -906.42413 \\
\hline
\end{tabular}

Table S5. Energies optimized for Figure 9b at $\omega B 97 X-D / 6-31 G(d, p)$ level (300K, $1 \mathrm{~atm})$

\begin{tabular}{cccc}
\hline & $\mathrm{B}^{\prime}$ & $\mathrm{TS}_{\mathrm{B}}{ }^{\prime} \mathrm{C}$ & $\mathrm{C}$ \\
\hline $\mathrm{E}$ (Hartree) & -831.27939 & -831.23184 & -831.24805 \\
$\mathrm{G}$ (Hartree) & -831.21432 & -831.16922 & -831.18477 \\
\hline
\end{tabular}

Table S6. Energies optimized for Figure 9c at $\omega$ B97X-D/6-31G (d,p) level (300K, $1 \mathrm{~atm})$

\begin{tabular}{cccc}
\hline & $\mathrm{C}^{\prime}$ & $\mathrm{TS}_{\mathrm{C}^{\prime}-\mathrm{D}}$ & $\mathrm{D}$ \\
\hline $\mathrm{E}$ (Hartree) & -757.32257 & -757.23491 & -757.40347 \\
$\mathrm{G}$ (Hartree) & -757.24583 & -757.16051 & -757.31827 \\
\hline
\end{tabular}

Table S7. Energies optimized for Figure 9d at $\omega B$ 97X-D/6-31G (d,p) level (300K, $1 \mathrm{~atm})$

\begin{tabular}{cccc}
\hline & $\mathrm{A}$ & $\mathrm{TS}_{\mathrm{A}-\mathrm{B}}$ & $\mathrm{B}$ \\
\hline $\mathrm{E}$ (Hartree) & -712.54021 & -712.50175 & -712.59683 \\
$\mathrm{G}$ (Hartree) & -712.47508 & -712.43764 & -712.53254 \\
\hline
\end{tabular}

Table S8. Energies optimized for Figure 9e at $\omega$ B97X-D/6-31G (d,p) level (300K, $1 \mathrm{~atm})$

\begin{tabular}{cccc}
\hline & $\mathrm{B}^{\prime}$ & $\mathrm{TS}_{\mathrm{B}}{ }^{\prime} \mathrm{C}$ & $\mathrm{C}$ \\
\hline $\mathrm{E}$ (Hartree) & -637.3511 & -637.31059 & -637.40465 \\
$\mathrm{G}$ (Hartree) & -637.2887 & -637.24932 & -637.34361 \\
\hline
\end{tabular}

Table S9. Energies optimized for Figure 9f at $\omega$ B97X-D/6-31G (d,p) level (300K, $1 \mathrm{~atm})$

\begin{tabular}{cccc}
\hline & $\mathrm{C}^{\prime}$ & $\mathrm{TS}_{\mathrm{C}^{\prime} \mathrm{D}}$ & $\mathrm{D}$ \\
\hline $\mathrm{E}$ (Hartree) & -563.35633 & -563.30811 & -563.42692 \\
$\mathrm{G}$ (Hartree) & -563.28412 & -563.23556 & -563.34353 \\
\hline
\end{tabular}


Table S10. Coordinates of structures optimized at $\omega$ B97X-D/6-31G $(d, p)$ level

A (Figure 9a)

$\begin{array}{cccc}\mathrm{C} & 1.688 & 1.206 & 0.432 \\ \mathrm{C} & 1.049 & -0.007 & 0.689 \\ \mathrm{C} & 1.691 & -1.222 & 0.435 \\ \mathrm{C} & 2.974 & -1.221 & -0.084 \\ \mathrm{C} & 3.621 & -0.01 & -0.349 \\ \mathrm{C} & 2.974 & 1.201 & -0.088 \\ \mathrm{H} & 1.176 & 2.136 & 0.644 \\ \mathrm{H} & 1.178 & -2.152 & 0.65 \\ \mathrm{H} & 3.493 & -2.15 & -0.293 \\ \mathrm{H} & 3.475 & 2.144 & -0.293 \\ \mathrm{O} & -0.914 & -1.1 & 1.234 \\ \mathrm{O} & -0.909 & 1.091 & 1.245 \\ \mathrm{O} & 4.874 & -0.076 & -0.855 \\ \mathrm{H} & 5.214 & 0.816 & -0.998 \\ \mathrm{~N} & -0.272 & -0.006 & 1.204 \\ \mathrm{Cu} & -2.086 & 1.188 & -0.463 \\ \mathrm{Cu} & -2.087 & -1.18 & -0.479\end{array}$

Transition state $\mathbf{T S}_{\text {A-B }}($ Figure 9a)

$\begin{array}{cccc}\mathrm{C} & 1.522 & -0.307 & 1.423 \\ \mathrm{C} & 0.69 & -0.855 & 0.448 \\ \mathrm{C} & 1.181 & -1.091 & -0.838 \\ \mathrm{C} & 2.491 & -0.757 & -1.154 \\ \mathrm{C} & 3.319 & -0.189 & -0.181 \\ \mathrm{C} & 2.832 & 0.035 & 1.111 \\ \mathrm{H} & 1.12 & -0.139 & 2.417 \\ \mathrm{H} & 0.523 & -1.546 & -1.569 \\ \mathrm{H} & 2.897 & -0.941 & -2.143 \\ \mathrm{H} & 3.479 & 0.465 & 1.872 \\ \mathrm{O} & -1.309 & -1.794 & -0.071 \\ \mathrm{O} & -1.423 & 0.52 & 0.788 \\ \mathrm{O} & 4.591 & 0.119 & -0.546 \\ \mathrm{H} & 5.067 & 0.467 & 0.217 \\ \mathrm{~N} & -0.655 & -1.184 & 0.852 \\ \mathrm{Cu} & -0.511 & 1.698 & -0.215 \\ \mathrm{Cu} & -2.785 & -0.381 & -0.232 \\ & & & \\ & \text { B (Figure 9a) } & \end{array}$

$\begin{array}{llll}\mathrm{C} & 1.378 & 0.135 & 1.214 \\ \mathrm{C} & -1.74 & 1.088 & 0.239 \\ \mathrm{C} & 2.096 & 0.684 & -1.045 \\ \mathrm{C} & 2.092 & -0.661 & -1.391 \\ \mathrm{C} & 1.734 & -1.62 & -0.443 \\ \mathrm{C} & 1.376 & -1.234 & 0.879 \\ \mathrm{H} & 1.247 & 0.465 & 2.242 \\ \mathrm{H} & 2.373 & 1.446 & -1.766\end{array}$




$\begin{array}{cccc}\mathrm{H} & 2.364 & -0.991 & -2.387 \\ \mathrm{H} & 1.289 & -1.993 & 1.655 \\ \mathrm{O} & 2.016 & 3.26 & -0.185 \\ \mathrm{O} & 2.346 & -0.647 & 0.591 \\ \mathrm{O} & 1.762 & -2.906 & -0.816 \\ \mathrm{H} & 1.409 & -3.47 & -0.115 \\ \mathrm{~N} & 1.728 & 2.451 & 0.673 \\ \mathrm{Cu} & 0.579 & -0.573 & 0.717\end{array}$

B' (Figure 9b)

$\begin{array}{cccc}\mathrm{C} & -0.126 & -0.381 & 0.415 \\ \mathrm{C} & -1.061 & 0.677 & 0.679 \\ \mathrm{C} & -0.643 & -1.624 & -0.056 \\ \mathrm{C} & -2.456 & 0.44 & 0.481 \\ \mathrm{H} & -0.786 & 1.409 & 1.442 \\ \mathrm{~N} & 1.202 & -0.167 & 0.539 \\ \mathrm{C} & -1.982 & -1.8 & -0.263 \\ \mathrm{H} & 0.054 & -2.43 & -0.279 \\ \mathrm{C} & -2.908 & -0.754 & -0.011 \\ \mathrm{H} & -3.157 & 1.228 & 0.75 \\ \mathrm{O} & 1.582 & 1.126 & 0.65 \\ \mathrm{H} & -2.365 & -2.744 & -0.639 \\ \mathrm{O} & -4.221 & -1.039 & -0.266 \\ \mathrm{Cu} & 2.535 & -1.247 & -0.133 \\ \mathrm{H} & -4.753 & -0.26 & -0.065 \\ \mathrm{Cu} & 0.18 & 2.072 & -0.402\end{array}$

Transition state $\mathbf{T S}_{\mathbf{B}}{ }^{-}-\mathbf{C}$ (Figure 9b)

\begin{tabular}{cccc}
$\mathrm{C}$ & -0.209 & -0.347 & 0.567 \\
$\mathrm{C}$ & -1.17 & 0.55 & 1.139 \\
$\mathrm{C}$ & -0.715 & -1.314 & -0.349 \\
$\mathrm{C}$ & -2.538 & 0.443 & 0.844 \\
$\mathrm{H}$ & -0.835 & 1.214 & 1.934 \\
$\mathrm{~N}$ & 1.113 & -0.221 & 0.985 \\
$\mathrm{C}$ & -2.058 & -1.402 & -0.644 \\
$\mathrm{H}$ & -0.007 & -1.998 & -0.807 \\
$\mathrm{C}$ & -2.981 & -0.524 & -0.05 \\
$\mathrm{H}$ & -3.247 & 1.106 & 1.335 \\
$\mathrm{O}$ & 1.907 & 0.757 & -0.582 \\
$\mathrm{H}$ & -2.432 & -2.152 & -1.334 \\
$\mathrm{O}$ & -4.285 & -0.685 & -0.387 \\
$\mathrm{Cu}$ & 2.569 & -0.88 & 0.062 \\
$\mathrm{H}$ & -4.823 & -0.039 & 0.087 \\
$\mathrm{Cu}$ & 0.21 & 1.514 & -0.386 \\
& \multicolumn{3}{c}{} \\
& \multicolumn{2}{c}{$\mathrm{C}$ (Figure $9 \mathrm{~b})$} & \\
$\mathrm{C}$ & -0.734 & 0.059 & 0 \\
$\mathrm{C}$ & -1.614 & 1.163 & 0 \\
$\mathrm{C}$ & -1.32 & -1.232 & -0.001
\end{tabular}




$\begin{array}{cccc}\mathrm{C} & -2.988 & 0.997 & 0 \\ \mathrm{H} & -1.195 & 2.167 & 0 \\ \mathrm{~N} & 0.631 & 0.197 & 0 \\ \mathrm{C} & -2.688 & -1.405 & 0 \\ \mathrm{H} & -0.665 & -2.099 & -0.001 \\ \mathrm{C} & -3.535 & -0.29 & 0 \\ \mathrm{H} & -3.64 & 1.868 & 0.001 \\ \mathrm{O} & 3.206 & -2.04 & 0.001 \\ \mathrm{H} & -3.128 & -2.397 & -0.001 \\ \mathrm{O} & -4.867 & -0.521 & 0 \\ \mathrm{Cu} & 1.93 & -0.984 & 0 \\ \mathrm{H} & -5.343 & 0.319 & 0.001 \\ \mathrm{Cu} & 1.523 & 1.794 & 0\end{array}$

C' (Figure 9c)

$\begin{array}{cccc}\mathrm{C} & 0.547 & 0.045 & 0.263 \\ \mathrm{C} & 1.43 & 0.996 & 0.806 \\ \mathrm{C} & 1.135 & -1.01 & -0.463 \\ \mathrm{C} & 2.806 & 0.901 & 0.642 \\ \mathrm{H} & 1.008 & 1.819 & 1.377 \\ \mathrm{~N} & -0.828 & 0.143 & 0.537 \\ \mathrm{C} & 2.508 & -1.12 & -0.627 \\ \mathrm{H} & 0.483 & -1.759 & -0.911 \\ \mathrm{C} & 3.356 & -0.161 & -0.075 \\ \mathrm{H} & 3.455 & 1.657 & 1.081 \\ \mathrm{H} & 2.94 & -1.943 & -1.188 \\ \mathrm{O} & 4.7 & -0.31 & -0.27 \\ \mathrm{H} & 5.159 & 0.416 & 0.17 \\ \mathrm{Cu} & -1.71 & 1.416 & -0.414 \\ \mathrm{Cu} & -2.052 & -1.143 & 0.22 \\ \mathrm{H} & -3.168 & -2.363 & 0.498 \\ \mathrm{H} & -3.283 & -2.166 & -0.261\end{array}$

Intermediate TSC'-D (Figure 9c)

$\begin{array}{cccc}\mathrm{C} & -1.272 & -0.535 & -0.294 \\ \mathrm{C} & -1.665 & 0.83 & -0.417 \\ \mathrm{C} & -2.282 & -1.464 & 0.099 \\ \mathrm{C} & -2.955 & 1.238 & -0.153 \\ \mathrm{H} & -0.917 & 1.548 & -0.745 \\ \mathrm{~N} & -0.027 & -0.978 & -0.558 \\ \mathrm{C} & -3.57 & -1.066 & 0.369 \\ \mathrm{H} & -1.991 & -2.506 & 0.188 \\ \mathrm{C} & -3.912 & 0.291 & 0.245 \\ \mathrm{H} & -3.237 & 2.283 & -0.259 \\ \mathrm{H} & -4.338 & -1.77 & 0.672 \\ \mathrm{O} & -5.186 & 0.626 & 0.514 \\ \mathrm{H} & -5.309 & 1.577 & 0.4 \\ \mathrm{Cu} & 1.56 & 0.018 & -0.575 \\ \mathrm{Cu} & 3.571 & 0.208 & 0.526 \\ \mathrm{H} & 0.923 & -1.008 & 0.811\end{array}$




\begin{tabular}{cccc}
$\mathrm{H}$ & 1.664 & -0.587 & 1.051 \\
& \multicolumn{3}{c}{ D (Figure 9c) } \\
\multicolumn{4}{c}{} \\
$\mathrm{C}$ & -1.331 & 0.901 & 0.191 \\
$\mathrm{C}$ & -1.823 & 0.15 & 1.252 \\
$\mathrm{C}$ & -2.008 & 0.898 & -1.027 \\
$\mathrm{C}$ & -2.994 & -0.587 & 1.105 \\
$\mathrm{H}$ & -1.289 & 0.128 & 2.198 \\
$\mathrm{~N}$ & -0.094 & 1.63 & 0.336 \\
$\mathrm{C}$ & -3.174 & 0.16 & -1.18 \\
$\mathrm{H}$ & -1.622 & 1.469 & -1.867 \\
$\mathrm{C}$ & -3.674 & -0.586 & -0.112 \\
$\mathrm{H}$ & -3.369 & -1.169 & 1.943 \\
$\mathrm{H}$ & -3.707 & 0.15 & -2.124 \\
$\mathrm{O}$ & -4.821 & -1.285 & -0.32 \\
$\mathrm{H}$ & -5.046 & -1.778 & 0.477 \\
$\mathrm{Cu}$ & 1.556 & 0.456 & 0.078 \\
$\mathrm{Cu}$ & 3.421 & -0.8 & -0.171 \\
$\mathrm{H}$ & -0.027 & 2.049 & 1.261 \\
$\mathrm{H}$ & -0.044 & 2.393 & -0.337
\end{tabular}

\section{A (Figure 9d)}

$\begin{array}{cccc}\mathrm{C} & -2.697 & 1.241 & 0.031 \\ \mathrm{C} & -1.854 & 0.124 & 0.034 \\ \mathrm{C} & -2.395 & -1.171 & 0.008 \\ \mathrm{C} & -3.767 & -1.338 & -0.02 \\ \mathrm{C} & -4.615 & -0.226 & -0.024 \\ \mathrm{C} & -4.071 & 1.061 & 0.002 \\ \mathrm{H} & -2.266 & 2.234 & 0.052 \\ \mathrm{H} & -1.731 & -2.025 & 0.011 \\ \mathrm{H} & -4.205 & -2.33 & -0.041 \\ \mathrm{H} & -4.724 & 1.93 & 0 \\ \mathrm{O} & 0.281 & -0.785 & 0.06 \\ \mathrm{O} & 0.023 & 1.49 & 0.083 \\ \mathrm{O} & -5.951 & -0.46 & -0.053 \\ \mathrm{H} & -6.424 & 0.381 & -0.051 \\ \mathrm{~N} & -0.468 & 0.31 & 0.062 \\ \mathrm{Cu} & 2.103 & 1.521 & -0.039 \\ \mathrm{Sn} & 2.464 & -0.931 & -0.004\end{array}$

Transition state $\mathbf{T S}_{\text {A-B }}$ (Figure 9d)

$\begin{array}{cccc}\mathrm{C} & 2.438 & 1.074 & -0.578 \\ \mathrm{C} & 1.734 & 0.299 & 0.341 \\ \mathrm{C} & 2.375 & -0.743 & 1.017 \\ \mathrm{C} & 3.71 & -1.013 & 0.777 \\ \mathrm{C} & 4.42 & -0.232 & -0.142 \\ \mathrm{C} & 3.781 & 0.812 & -0.816 \\ \mathrm{H} & 1.932 & 1.881 & -1.093 \\ \mathrm{H} & 1.8 & -1.342 & 1.714\end{array}$




$\begin{array}{cccc}\mathrm{H} & 4.224 & -1.82 & 1.287 \\ \mathrm{H} & 4.335 & 1.421 & -1.527 \\ \mathrm{O} & -0.385 & -1 & 0.063 \\ \mathrm{O} & -0.113 & 1.557 & 0.054 \\ \mathrm{O} & 5.729 & -0.533 & -0.333 \\ \mathrm{H} & 6.101 & 0.067 & -0.99 \\ \mathrm{~N} & 0.378 & 0.55 & 0.692 \\ \mathrm{Cu} & -2.046 & 1.562 & 0.196 \\ \mathrm{Sn} & -2.286 & -1.015 & -0.236\end{array}$

Product B (Figure 9d)

$\begin{array}{cccc}\mathrm{C} & -2.401 & 1.407 & 0 \\ \mathrm{C} & -1.52 & 0.313 & 0 \\ \mathrm{C} & -2.056 & -0.987 & 0 \\ \mathrm{C} & -3.424 & -1.191 & 0 \\ \mathrm{C} & -4.293 & -0.095 & 0 \\ \mathrm{C} & -3.771 & 1.201 & 0 \\ \mathrm{H} & -1.99 & 2.408 & 0 \\ \mathrm{H} & -1.396 & -1.85 & 0 \\ \mathrm{H} & -3.841 & -2.192 & 0 \\ \mathrm{H} & -4.443 & 2.057 & 0 \\ \mathrm{O} & 3.066 & -0.082 & 0 \\ \mathrm{O} & 0.263 & 1.732 & 0 \\ \mathrm{O} & -5.626 & -0.355 & 0 \\ \mathrm{H} & -6.113 & 0.478 & 0 \\ \mathrm{~N} & -0.136 & 0.479 & 0 \\ \mathrm{Cu} & 2.199 & 1.562 & 0 \\ \mathrm{Sn} & 1.563 & -1.276 & 0\end{array}$

B' (Figure 9e)

$\begin{array}{cccc}\mathrm{C} & -2.193 & -1.308 & -0.005 \\ \mathrm{C} & -1.556 & -0.056 & -0.013 \\ \mathrm{C} & -2.339 & 1.112 & -0.011 \\ \mathrm{C} & -3.719 & 1.036 & -0.001 \\ \mathrm{C} & -4.348 & -0.214 & 0.006 \\ \mathrm{C} & -3.577 & -1.38 & 0.004 \\ \mathrm{H} & -1.588 & -2.206 & -0.007 \\ \mathrm{H} & -1.847 & 2.082 & -0.017 \\ \mathrm{H} & -4.331 & 1.932 & 0.001 \\ \mathrm{H} & -4.064 & -2.353 & 0.01 \\ \mathrm{O} & 0.508 & -1.045 & -0.025 \\ \mathrm{O} & -5.706 & -0.228 & 0.016 \\ \mathrm{H} & -6.016 & -1.142 & 0.02 \\ \mathrm{~N} & -0.18 & 0.078 & -0.023 \\ \mathrm{Cu} & 1.103 & 1.547 & 0.003 \\ \mathrm{Sn} & 2.702 & -0.574 & 0.005\end{array}$

Transition state $\mathbf{T S}_{\mathbf{B}}{ }^{-\mathbf{C}}$ (Figure 9e)

$\begin{array}{llll}\text { C } & 1.826 & 1.197 & 0.039\end{array}$ 


$\begin{array}{cccc}\mathrm{C} & 0.963 & 0.133 & -0.299 \\ \mathrm{C} & 1.573 & -1.138 & -0.439 \\ \mathrm{C} & 2.933 & -1.33 & -0.264 \\ \mathrm{C} & 3.755 & -0.254 & 0.074 \\ \mathrm{C} & 3.189 & 1.012 & 0.222 \\ \mathrm{H} & 1.403 & 2.193 & 0.158 \\ \mathrm{H} & 0.963 & -1.993 & -0.728 \\ \mathrm{H} & 3.378 & -2.312 & -0.387 \\ \mathrm{H} & 3.817 & 1.863 & 0.483 \\ \mathrm{O} & -1.483 & 0.477 & 1.043 \\ \mathrm{O} & 5.088 & -0.499 & 0.241 \\ \mathrm{H} & 5.53 & 0.325 & 0.475 \\ \mathrm{~N} & -0.397 & 0.329 & -0.468 \\ \mathrm{Cu} & -1.263 & 2.016 & -0.169 \\ \mathrm{Sn} & -1.799 & -1.168 & 0.038\end{array}$

C (Figure 9e)

$\begin{array}{cccc}\mathrm{C} & -2.169 & 1.273 & 0 \\ \mathrm{C} & -1.273 & 0.159 & 0 \\ \mathrm{C} & -1.873 & -1.143 & 0 \\ \mathrm{C} & -3.238 & -1.312 & 0 \\ \mathrm{C} & -4.084 & -0.19 & 0 \\ \mathrm{C} & -3.537 & 1.103 & 0 \\ \mathrm{H} & -1.734 & 2.268 & 0 \\ \mathrm{H} & -1.222 & -2.013 & 0 \\ \mathrm{H} & -3.686 & -2.3 & 0 \\ \mathrm{H} & -4.194 & 1.97 & 0 \\ \mathrm{O} & 2.933 & 0.548 & 0 \\ \mathrm{O} & -5.415 & -0.42 & 0 \\ \mathrm{H} & -5.891 & 0.421 & 0 \\ \mathrm{~N} & 0.048 & 0.338 & 0 \\ \mathrm{Cu} & 1.39 & 1.721 & 0 \\ \mathrm{Sn} & 1.86 & -1.06 & 0\end{array}$

C' (Figure 9f)

$\begin{array}{cccc}\mathrm{C} & 2.026 & 1.272 & 0.265 \\ \mathrm{C} & 1.102 & 0.184 & 0.296 \\ \mathrm{C} & 1.651 & -1.127 & 0.14 \\ \mathrm{C} & 3 & -1.328 & -0.045 \\ \mathrm{C} & 3.875 & -0.231 & -0.082 \\ \mathrm{C} & 3.377 & 1.071 & 0.076 \\ \mathrm{H} & 1.63 & 2.275 & 0.401 \\ \mathrm{H} & 0.977 & -1.978 & 0.171 \\ \mathrm{H} & 3.411 & -2.325 & -0.163 \\ \mathrm{H} & 4.058 & 1.92 & 0.055 \\ \mathrm{O} & 5.19 & -0.492 & -0.269 \\ \mathrm{H} & 5.687 & 0.335 & -0.271 \\ \mathrm{~N} & -0.205 & 0.398 & 0.506 \\ \mathrm{Cu} & -1.486 & 1.667 & -0.145 \\ \mathrm{H} & -1.297 & -2.254 & -0.846\end{array}$




$$
\begin{array}{cccc}
\mathrm{H} & -2.417 & -1.556 & 1.508 \\
\mathrm{Sn} & -1.985 & -0.853 & -0.039
\end{array}
$$

Intermediate TSC'-D (Figure 9f)

$\begin{array}{cccc}\mathrm{C} & 2.311 & 1.07 & -0.151 \\ \mathrm{C} & 1.596 & 0.011 & 0.494 \\ \mathrm{C} & 2.336 & -1.197 & 0.722 \\ \mathrm{C} & 3.652 & -1.333 & 0.344 \\ \mathrm{C} & 4.311 & -0.266 & -0.288 \\ \mathrm{C} & 3.63 & 0.935 & -0.531 \\ \mathrm{H} & 1.784 & 2.003 & -0.336 \\ \mathrm{H} & 1.819 & -2.015 & 1.215 \\ \mathrm{H} & 4.201 & -2.252 & 0.521 \\ \mathrm{H} & 4.145 & 1.761 & -1.019 \\ \mathrm{O} & 5.605 & -0.457 & -0.64 \\ \mathrm{H} & 5.945 & 0.343 & -1.061 \\ \mathrm{~N} & 0.333 & 0.09 & 0.928 \\ \mathrm{Cu} & -0.968 & 1.22 & 0.307 \\ \mathrm{H} & -0.773 & -1.465 & 0.963 \\ \mathrm{H} & -1.406 & -1.93 & 0.912 \\ \mathrm{Sn} & -2.837 & -0.482 & -0.3\end{array}$

D (Figure 9f)

$\begin{array}{cccc}\mathrm{C} & 1.701 & 1.011 & 0.506 \\ \mathrm{C} & 1.408 & -0.307 & 0.843 \\ \mathrm{C} & 2.348 & -1.305 & 0.592 \\ \mathrm{C} & 3.573 & -0.99 & 0.022 \\ \mathrm{C} & 3.87 & 0.332 & -0.307 \\ \mathrm{C} & 2.929 & 1.331 & -0.061 \\ \mathrm{H} & 0.96 & 1.79 & 0.672 \\ \mathrm{H} & 2.122 & -2.34 & 0.838 \\ \mathrm{H} & 4.311 & -1.76 & -0.176 \\ \mathrm{H} & 3.146 & 2.365 & -0.318 \\ \mathrm{O} & 5.087 & 0.582 & -0.86 \\ \mathrm{H} & 5.159 & 1.522 & -1.064 \\ \mathrm{~N} & 0.119 & -0.642 & 1.379 \\ \mathrm{Cu} & -2.326 & 1.518 & 0.08 \\ \mathrm{H} & 0.154 & -1.507 & 1.911 \\ \mathrm{H} & -0.232 & 0.088 & 1.993 \\ \mathrm{Sn} & -1.693 & -0.895 & -0.37\end{array}$


Table S11. Charge of A on $\mathrm{Cu}-\mathrm{Cu}$

\begin{tabular}{ccc}
\hline Atom & Number & Charge \\
\hline $\mathrm{C}$ & 1 & -0.205310 \\
$\mathrm{C}$ & 2 & 0.047860 \\
$\mathrm{C}$ & 3 & -0.207760 \\
$\mathrm{C}$ & 4 & -0.288830 \\
$\mathrm{C}$ & 5 & 0.355500 \\
$\mathrm{C}$ & 6 & -0.321840 \\
$\mathrm{H}$ & 7 & 0.274200 \\
$\mathrm{H}$ & 8 & 0.274470 \\
$\mathrm{H}$ & 9 & 0.262710 \\
$\mathrm{H}$ & 10 & 0.244460 \\
$\mathrm{O}$ & 11 & -0.521360 \\
$\mathrm{O}$ & 12 & -0.522990 \\
$\mathrm{O}$ & 13 & -0.685700 \\
$\mathrm{H}$ & 14 & 0.495730 \\
$\mathrm{~N}$ & 15 & 0.464510 \\
$\mathrm{Cu}$ & 16 & 0.164570 \\
$\mathrm{Cu}$ & 17 & 0.169770 \\
\hline
\end{tabular}

Table S12. Charge of $\mathrm{TS}_{\mathrm{A}-\mathrm{B}}$ on $\mathrm{Cu}-\mathrm{Cu}$

\begin{tabular}{ccc}
\hline Atom & Number & Charge \\
\hline $\mathrm{C}$ & 1 & -0.20582 \\
$\mathrm{C}$ & 2 & 0.05037 \\
$\mathrm{C}$ & 3 & -0.23043 \\
$\mathrm{C}$ & 4 & -0.29919 \\
$\mathrm{C}$ & 5 & 0.3393 \\
$\mathrm{C}$ & 6 & -0.33349 \\
$\mathrm{H}$ & 7 & 0.26647 \\
$\mathrm{H}$ & 8 & 0.26923 \\
$\mathrm{H}$ & 9 & 0.25684 \\
$\mathrm{H}$ & 10 & 0.24019 \\
$\mathrm{O}$ & 11 & -0.58501 \\
$\mathrm{O}$ & 12 & -0.93106 \\
$\mathrm{O}$ & 13 & -0.69391 \\
$\mathrm{H}$ & 14 & 0.49247 \\
$\mathrm{~N}$ & 15 & 0.12699 \\
$\mathrm{Cu}$ & 16 & 0.57437 \\
$\mathrm{Cu}$ & 17 & 0.66266 \\
\hline
\end{tabular}


Table S13. Charge of B on $\mathrm{Cu}-\mathrm{Cu}$

\begin{tabular}{ccc}
\hline Atom & Number & Charge \\
\hline $\mathrm{C}$ & 1 & -0.28357 \\
$\mathrm{C}$ & 2 & 0.01331 \\
$\mathrm{C}$ & 3 & -0.16651 \\
$\mathrm{C}$ & 4 & -0.30018 \\
$\mathrm{C}$ & 5 & 0.40686 \\
$\mathrm{C}$ & 6 & -0.44439 \\
$\mathrm{H}$ & 7 & 0.2969 \\
$\mathrm{H}$ & 8 & 0.27421 \\
$\mathrm{H}$ & 9 & 0.27064 \\
$\mathrm{H}$ & 10 & 0.28253 \\
$\mathrm{O}$ & 11 & -0.3045 \\
$\mathrm{O}$ & 12 & -0.99763 \\
$\mathrm{O}$ & 13 & -0.66674 \\
$\mathrm{H}$ & 14 & 0.50834 \\
$\mathrm{~N}$ & 15 & 0.10066 \\
$\mathrm{Cu}$ & 16 & 0.47245 \\
$\mathrm{Cu}$ & 17 & 0.53763 \\
\hline
\end{tabular}

Table S14. Charge of B' on $\mathrm{Cu}-\mathrm{Cu}$

\begin{tabular}{ccc}
\hline Atom & Number & Charge \\
\hline $\mathrm{C}$ & 1 & 0.15817 \\
$\mathrm{C}$ & 2 & -0.52365 \\
$\mathrm{C}$ & 3 & -0.2534 \\
$\mathrm{C}$ & 4 & -0.28618 \\
$\mathrm{H}$ & 5 & 0.27915 \\
$\mathrm{~N}$ & 6 & -0.34297 \\
$\mathrm{C}$ & 7 & -0.25128 \\
$\mathrm{H}$ & 8 & 0.23168 \\
$\mathrm{C}$ & 9 & 0.28712 \\
$\mathrm{H}$ & 10 & 0.23629 \\
$\mathrm{O}$ & 11 & -0.64308 \\
$\mathrm{H}$ & 12 & 0.25452 \\
$\mathrm{O}$ & 13 & -0.70649 \\
$\mathrm{Cu}$ & 14 & 0.55978 \\
$\mathrm{H}$ & 15 & 0.4888 \\
$\mathrm{Cu}$ & 16 & 0.51155 \\
\hline
\end{tabular}


Table S15. Charge of $\mathrm{TS}_{\mathrm{B}}{ }^{-} \mathrm{C}$ on $\mathrm{Cu}-\mathrm{Cu}$

\begin{tabular}{ccc}
\hline Atom & Number & Charge \\
\hline $\mathrm{C}$ & 1 & 0.03436 \\
$\mathrm{C}$ & 2 & -0.35273 \\
$\mathrm{C}$ & 3 & -0.20654 \\
$\mathrm{C}$ & 4 & -0.31125 \\
$\mathrm{H}$ & 5 & 0.2812 \\
$\mathrm{~N}$ & 6 & -0.63018 \\
$\mathrm{C}$ & 7 & -0.27281 \\
$\mathrm{H}$ & 8 & 0.25167 \\
$\mathrm{C}$ & 9 & 0.33621 \\
$\mathrm{H}$ & 10 & 0.24527 \\
$\mathrm{O}$ & 11 & -0.85878 \\
$\mathrm{H}$ & 12 & 0.26123 \\
$\mathrm{O}$ & 13 & -0.69057 \\
$\mathrm{Cu}$ & 14 & 0.72045 \\
$\mathrm{H}$ & 15 & 0.49433 \\
$\mathrm{Cu}$ & 16 & 0.69813 \\
\hline
\end{tabular}

Table S16. Charge of $\mathrm{C}$ on $\mathrm{Cu}-\mathrm{Cu}$

\begin{tabular}{ccc}
\hline Atom & Number & Charge \\
\hline $\mathrm{C}$ & 1 & 0.11943 \\
$\mathrm{C}$ & 2 & -0.20431 \\
$\mathrm{C}$ & 3 & -0.19217 \\
$\mathrm{C}$ & 4 & -0.32834 \\
$\mathrm{H}$ & 5 & 0.23132 \\
$\mathrm{~N}$ & 6 & -0.87926 \\
$\mathrm{C}$ & 7 & -0.29292 \\
$\mathrm{H}$ & 8 & 0.25721 \\
$\mathrm{C}$ & 9 & 0.35969 \\
$\mathrm{H}$ & 10 & 0.24232 \\
$\mathrm{O}$ & 11 & -0.62251 \\
$\mathrm{H}$ & 12 & 0.26369 \\
$\mathrm{O}$ & 13 & -0.68114 \\
$\mathrm{Cu}$ & 14 & 0.5967 \\
$\mathrm{H}$ & 15 & 0.4956 \\
$\mathrm{Cu}$ & 16 & 0.63466 \\
\hline
\end{tabular}


Table S17. Charge of C' on $\mathrm{Cu}-\mathrm{Cu}$

\begin{tabular}{ccc}
\hline Atom & Number & Charge \\
\hline $\mathrm{C}$ & 1 & 0.13265 \\
$\mathrm{C}$ & 2 & -0.23562 \\
$\mathrm{C}$ & 3 & -0.24415 \\
$\mathrm{C}$ & 4 & -0.32315 \\
$\mathrm{H}$ & 5 & 0.24317 \\
$\mathrm{~N}$ & 6 & -1.0194 \\
$\mathrm{C}$ & 7 & -0.29217 \\
$\mathrm{H}$ & 8 & 0.22911 \\
$\mathrm{C}$ & 9 & 0.31328 \\
$\mathrm{H}$ & 10 & 0.23113 \\
$\mathrm{H}$ & 11 & 0.24923 \\
$\mathrm{O}$ & 12 & -0.70383 \\
$\mathrm{H}$ & 13 & 0.48693 \\
$\mathrm{Cu}$ & 14 & 0.46013 \\
$\mathrm{Cu}$ & 15 & 0.39011 \\
$\mathrm{H}$ & 16 & 0.04236 \\
\hline
\end{tabular}

Table S18. Charge of $\mathrm{TS}_{\mathrm{C}^{\prime}-\mathrm{D}}$ on $\mathrm{Cu}-\mathrm{Cu}$

\begin{tabular}{ccc}
\hline Atom & Number & Charge \\
\hline $\mathrm{C}$ & 1 & 0.07293 \\
$\mathrm{C}$ & 2 & -0.15297 \\
$\mathrm{C}$ & 3 & -0.14683 \\
$\mathrm{C}$ & 4 & -0.34811 \\
$\mathrm{H}$ & 5 & 0.25332 \\
$\mathrm{~N}$ & 6 & -0.64503 \\
$\mathrm{C}$ & 7 & -0.31653 \\
$\mathrm{H}$ & 8 & 0.26134 \\
$\mathrm{C}$ & 9 & 0.39794 \\
$\mathrm{H}$ & 10 & 0.24502 \\
$\mathrm{H}$ & 11 & 0.26307 \\
$\mathrm{O}$ & 12 & -0.67119 \\
$\mathrm{H}$ & 13 & 0.49977 \\
$\mathrm{Cu}$ & 14 & 0.23646 \\
$\mathrm{Cu}$ & 15 & -0.00788 \\
$\mathrm{H}$ & 16 & 0.09054 \\
\hline
\end{tabular}


Table S19. Charge of D on $\mathrm{Cu}-\mathrm{Cu}$

\begin{tabular}{ccc}
\hline Atom & Number & Charge \\
\hline $\mathrm{C}$ & 1 & 0.11803 \\
$\mathrm{C}$ & 2 & -0.24915 \\
$\mathrm{C}$ & 3 & -0.25221 \\
$\mathrm{C}$ & 4 & -0.3054 \\
$\mathrm{H}$ & 5 & 0.25132 \\
$\mathrm{~N}$ & 6 & -0.93396 \\
$\mathrm{C}$ & 7 & -0.27284 \\
$\mathrm{H}$ & 8 & 0.24808 \\
$\mathrm{C}$ & 9 & 0.32787 \\
$\mathrm{H}$ & 10 & 0.24318 \\
$\mathrm{H}$ & 11 & 0.2608 \\
$\mathrm{O}$ & 12 & -0.69453 \\
$\mathrm{H}$ & 13 & 0.49311 \\
$\mathrm{Cu}$ & 14 & 0.09022 \\
$\mathrm{Cu}$ & 15 & -0.18226 \\
$\mathrm{H}$ & 16 & 0.42957 \\
\hline
\end{tabular}

Table S20. Charge of A on $\mathrm{Cu}-\mathrm{Sn}$

\begin{tabular}{ccc}
\hline Atom & Number & Charge \\
\hline $\mathrm{C}$ & 1 & -0.21872 \\
$\mathrm{C}$ & 2 & 0.06244 \\
$\mathrm{C}$ & 3 & -0.21999 \\
$\mathrm{C}$ & 4 & -0.28832 \\
$\mathrm{C}$ & 5 & 0.34019 \\
$\mathrm{C}$ & 6 & -0.32041 \\
$\mathrm{H}$ & 7 & 0.27065 \\
$\mathrm{H}$ & 8 & 0.27274 \\
$\mathrm{H}$ & 9 & 0.25951 \\
$\mathrm{H}$ & 10 & 0.24121 \\
$\mathrm{O}$ & 11 & -0.67907 \\
$\mathrm{O}$ & 12 & -0.57027 \\
$\mathrm{O}$ & 13 & -0.69077 \\
$\mathrm{H}$ & 14 & 0.4932 \\
$\mathrm{~N}$ & 15 & 0.4269 \\
$\mathrm{Cu}$ & 16 & 0.08065 \\
$\mathrm{Sn}$ & 17 & 0.54006 \\
\hline
\end{tabular}


Table S21. Charge of $\mathrm{TS}_{\mathrm{A}-\mathrm{B}}$ on $\mathrm{Cu}-\mathrm{Sn}$

\begin{tabular}{ccc}
\hline Atom & Number & Charge \\
\hline $\mathrm{C}$ & 1 & -0.2086 \\
$\mathrm{C}$ & 2 & 0.07124 \\
$\mathrm{C}$ & 3 & -0.18275 \\
$\mathrm{C}$ & 4 & -0.29998 \\
$\mathrm{C}$ & 5 & 0.35049 \\
$\mathrm{C}$ & 6 & -0.3299 \\
$\mathrm{H}$ & 7 & 0.26301 \\
$\mathrm{H}$ & 8 & 0.2629 \\
$\mathrm{H}$ & 9 & 0.2565 \\
$\mathrm{H}$ & 10 & 0.237 \\
$\mathrm{O}$ & 11 & -0.90031 \\
$\mathrm{O}$ & 12 & -0.56253 \\
$\mathrm{O}$ & 13 & -0.69141 \\
$\mathrm{H}$ & 14 & 0.49198 \\
$\mathrm{~N}$ & 15 & 0.12374 \\
$\mathrm{Cu}$ & 16 & 0.29957 \\
$\mathrm{Sn}$ & 17 & 0.81904 \\
\hline
\end{tabular}

Table S22. Charge of B on $\mathrm{Cu}-\mathrm{Sn}$

\begin{tabular}{ccc}
\hline Atom & Number & Charge \\
\hline $\mathrm{C}$ & 1 & -0.22149 \\
$\mathrm{C}$ & 2 & 0.0773 \\
$\mathrm{C}$ & 3 & -0.22931 \\
$\mathrm{C}$ & 4 & -0.28967 \\
$\mathrm{C}$ & 5 & 0.33812 \\
$\mathrm{C}$ & 6 & -0.31667 \\
$\mathrm{H}$ & 7 & 0.26921 \\
$\mathrm{H}$ & 8 & 0.23578 \\
$\mathrm{H}$ & 9 & 0.25793 \\
$\mathrm{H}$ & 10 & 0.23999 \\
$\mathrm{O}$ & 11 & -1.17419 \\
$\mathrm{O}$ & 12 & -0.55156 \\
$\mathrm{O}$ & 13 & -0.69196 \\
$\mathrm{H}$ & 14 & 0.49317 \\
$\mathrm{~N}$ & 15 & -0.24474 \\
$\mathrm{Cu}$ & 16 & 0.60198 \\
$\mathrm{Sn}$ & 17 & 1.20613 \\
\hline
\end{tabular}


Table S23. Charge of B' on Cu-Sn

\begin{tabular}{ccc}
\hline Atom & Number & Charge \\
\hline $\mathrm{C}$ & 1 & -0.22408 \\
$\mathrm{C}$ & 2 & 0.08665 \\
$\mathrm{C}$ & 3 & -0.22106 \\
$\mathrm{C}$ & 4 & -0.29068 \\
$\mathrm{C}$ & 5 & 0.33612 \\
$\mathrm{C}$ & 6 & -0.31612 \\
$\mathrm{H}$ & 7 & 0.26766 \\
$\mathrm{H}$ & 8 & 0.23898 \\
$\mathrm{H}$ & 9 & 0.2577 \\
$\mathrm{H}$ & 10 & 0.23968 \\
$\mathrm{O}$ & 11 & -0.57815 \\
$\mathrm{O}$ & 12 & -0.69263 \\
$\mathrm{H}$ & 13 & 0.49287 \\
$\mathrm{~N}$ & 14 & -0.2303 \\
$\mathrm{Cu}$ & 15 & 0.32033 \\
$\mathrm{Sn}$ & 16 & 0.31304 \\
\hline
\end{tabular}

Table S24. Charge of $\mathrm{TS}_{\mathrm{B}}{ }^{-}-\mathrm{C}$ on $\mathrm{Cu}-\mathrm{Sn}$

\begin{tabular}{ccc}
\hline Atom & Number & Charge \\
\hline $\mathrm{C}$ & 1 & -0.23426 \\
$\mathrm{C}$ & 2 & 0.12809 \\
$\mathrm{C}$ & 3 & -0.25896 \\
$\mathrm{C}$ & 4 & -0.28411 \\
$\mathrm{C}$ & 5 & 0.31124 \\
$\mathrm{C}$ & 6 & -0.31551 \\
$\mathrm{H}$ & 7 & 0.22312 \\
$\mathrm{H}$ & 8 & 0.2212 \\
$\mathrm{H}$ & 9 & 0.2515 \\
$\mathrm{H}$ & 10 & 0.23341 \\
$\mathrm{O}$ & 11 & -1.00526 \\
$\mathrm{O}$ & 12 & -0.7023 \\
$\mathrm{H}$ & 13 & 0.48793 \\
$\mathrm{~N}$ & 14 & -0.94432 \\
$\mathrm{Cu}$ & 15 & 0.67613 \\
$\mathrm{Sn}$ & 16 & 1.2121 \\
\hline
\end{tabular}


Table S25. Charge of $\mathrm{C}$ on $\mathrm{Cu}-\mathrm{Sn}$

\begin{tabular}{ccc}
\hline Atom & Number & Charge \\
\hline $\mathrm{C}$ & 1 & -0.18596 \\
$\mathrm{C}$ & 2 & 0.13086 \\
$\mathrm{C}$ & 3 & -0.20387 \\
$\mathrm{C}$ & 4 & -0.29635 \\
$\mathrm{C}$ & 5 & 0.36262 \\
$\mathrm{C}$ & 6 & -0.33117 \\
$\mathrm{H}$ & 7 & 0.24822 \\
$\mathrm{H}$ & 8 & 0.24287 \\
$\mathrm{H}$ & 9 & 0.26064 \\
$\mathrm{H}$ & 10 & 0.24247 \\
$\mathrm{O}$ & 11 & -1.15673 \\
$\mathrm{O}$ & 12 & -0.68004 \\
$\mathrm{H}$ & 13 & 0.4968 \\
$\mathrm{~N}$ & 14 & -0.89257 \\
$\mathrm{Cu}$ & 15 & 0.57408 \\
$\mathrm{Sn}$ & 16 & 1.18812 \\
\hline
\end{tabular}

Table S26. Charge of C' on $\mathrm{Cu}-\mathrm{Sn}$

\begin{tabular}{ccc}
\hline Atom & Number & Charge \\
\hline $\mathrm{C}$ & 1 & -0.19242 \\
$\mathrm{C}$ & 2 & 0.12881 \\
$\mathrm{C}$ & 3 & -0.2025 \\
$\mathrm{C}$ & 4 & -0.2953 \\
$\mathrm{C}$ & 5 & 0.35839 \\
$\mathrm{C}$ & 6 & -0.33213 \\
$\mathrm{H}$ & 7 & 0.24519 \\
$\mathrm{H}$ & 8 & 0.25377 \\
$\mathrm{H}$ & 9 & 0.25995 \\
$\mathrm{H}$ & 10 & 0.2406 \\
$\mathrm{O}$ & 11 & -0.6824 \\
$\mathrm{H}$ & 12 & 0.49485 \\
$\mathrm{~N}$ & 13 & -0.85652 \\
$\mathrm{Cu}$ & 14 & 0.48116 \\
$\mathrm{H}$ & 15 & -0.2721 \\
$\mathrm{H}$ & 16 & -0.25914 \\
\hline
\end{tabular}


Table S27. Charge of $\mathrm{TS}_{\mathrm{C}^{\prime}-\mathrm{D}}$ on $\mathrm{Cu}-\mathrm{Sn}$

\begin{tabular}{ccc}
\hline Atom & Number & Charge \\
\hline $\mathrm{C}$ & 1 & -0.19819 \\
$\mathrm{C}$ & 2 & 0.1034 \\
$\mathrm{C}$ & 3 & -0.19252 \\
$\mathrm{C}$ & 4 & -0.30467 \\
$\mathrm{C}$ & 5 & 0.3542 \\
$\mathrm{C}$ & 6 & -0.33606 \\
$\mathrm{H}$ & 7 & 0.2369 \\
$\mathrm{H}$ & 8 & 0.24872 \\
$\mathrm{H}$ & 9 & 0.25573 \\
$\mathrm{H}$ & 10 & 0.23727 \\
$\mathrm{O}$ & 11 & -0.68659 \\
$\mathrm{H}$ & 12 & 0.49303 \\
$\mathrm{~N}$ & 13 & -0.74183 \\
$\mathrm{Cu}$ & 14 & 0.23118 \\
$\mathrm{H}$ & 15 & 0.10203 \\
$\mathrm{H}$ & 16 & -0.11583 \\
\hline
\end{tabular}

Table S28. Charge of D on $\mathrm{Cu}-\mathrm{Sn}$

\begin{tabular}{ccc}
\hline Atom & Number & Charge \\
\hline $\mathrm{C}$ & 1 & -0.24387 \\
$\mathrm{C}$ & 2 & 0.11659 \\
$\mathrm{C}$ & 3 & -0.25316 \\
$\mathrm{C}$ & 4 & -0.276 \\
$\mathrm{C}$ & 5 & 0.32753 \\
$\mathrm{C}$ & 6 & -0.3067 \\
$\mathrm{H}$ & 7 & 0.25311 \\
$\mathrm{H}$ & 8 & 0.24594 \\
$\mathrm{H}$ & 9 & 0.25949 \\
$\mathrm{H}$ & 10 & 0.2422 \\
$\mathrm{O}$ & 11 & -0.6957 \\
$\mathrm{H}$ & 12 & 0.4926 \\
$\mathrm{~N}$ & 13 & -0.90542 \\
$\mathrm{Cu}$ & 14 & -0.18489 \\
$\mathrm{H}$ & 15 & 0.42547 \\
$\mathrm{H}$ & 16 & 0.43542 \\
\hline
\end{tabular}




\section{Reference}

(1) Gawande, M. B.; Rathi, A. K.; Branco, P. S.; Nogueira, I. D.; Velhinho, A.; Shrikhande, J. J.; Indulkar, U. U.; Jayaram, R. V.; Ghumman, C. A. A.; Bundaleski, N.; Teodoro, O. M. Regio- and chemoselective reduction of nitroarenes and carbonyl compounds over recyclable magnetic ferrite nickel nanoparticles $\left(\mathrm{Fe}_{3} \mathrm{O}_{4}-\mathrm{Ni}\right)$ by using glycerol as a hydrogen source. Chem. Eur. J. 2012, 18, $12628-12632$.

(2) Chu, C. S.; Rao, S.; Ma, Z. F.; Han, H. L. Copper and cobalt nanoparticles doped nitrogencontaining carbon frameworks derived from CuO-encapsulated ZIF-67 as high-efficiency catalyst for hydrogenation of 4-nitrophenol. Appl. Catal. B: Environ. 2019,256,117792.

(3) Yang, X. L.; Zhong, H.; Zhu, Y. H.; Jiang, H. L.; Shen, J. H.; Huang, J. F.; Li, C. Z. Highly efficient reusable catalyst based on silicon nanowire arrays decorated with copper nanoparticles. J. Mater. Chem. A 2014, 2, 9040-9047.

(4) Ye, W. C.; Yu, J.; Zhou, Y. X.; Gao, D. Q.; Wang, D. A.; Wang, C. M.; Xue, D. S. Green synthesis of $\mathrm{Pt}-\mathrm{Au}$ dendrimer-like nanoparticles supported on polydopamine-functionalized graphene and their high performance toward 4-nitrophenol reduction. Appl. Catal. B: Environ. 2016, 181, 371-378.

(5) Sudhakara, M.; Naresha, G.; Rambabu, G.; Anjaneyulu, C.; Padmasri, A. H.; Kantama, M. L.; Venugopala A. Crude bio-glycerol as a hydrogen source for the selective hydrogenation of aromatic nitro compounds over Ru/MgLaO catalyst. Catal. Commun. 2016, 74, 91-94.

(6) Frisch, M. J.; Trucks, G. W.; Schlegel, H. B.; Scuseria, G. E.; Robb, M. A.; Cheeseman, J. R.; Scalmani, G.; Barone, V.; Petersson, G. A.; Nakatsuji, H.; Li, X.; Caricato, M.; Marenich, A. V.; Bloino, J.; Janesko, B. G.; Gomperts, R.; Mennucci, B.; Hratchian, H. P.; Ortiz, J. V.; Izmaylov, A. F.; Sonnenberg, J. L.; Williams-Young, D.; Ding, F.; Lipparini, F.; Egidi, F.; Goings, J.; Peng, B.; Petrone, A.; Henderson, T.; Ranasinghe, D.; Zakrzewski, V. G.; Gao, J.; Rega, N.; Zheng, G.; Liang, W.; Hada, M.; Ehara, M.; Toyota, K.; Fukuda, R.; Hasegawa, J.; Ishida, M.; Nakajima, T.; Honda, Y.; Kitao, O.; Nakai, H.; Vreven, T.; Throssell, K.; Montgomery, J. A.; Peralta, J. E.; Ogliaro, F.; Bearpark, M. J.; Heyd, J. J.; Brothers, E. N.; Kudin, K. N.; Staroverov, V. N.; Keith, T. A.; Kobayashi, R.; Normand, J.; Raghavachari, K.; Rendell, A. P.; Burant, J. C.; Iyengar, S. S.; Tomasi, J.; Cossi, M.; Millam, J. M.; Klene, M.; Adamo, C.; Cammi, R.; Ochterski, J. W.; Martin, R. L.; Morokuma, K.; Farkas, O.; Foresman, J. B.; Fox, D. J. Gaussian 16, Revision A. 03. Gaussian, Inc.: Wallingford CT, 2016.

(7) Chai, J. D.; Head-Gordon, M. Long-range corrected hybrid density functionals with damped atomatom dispersion corrections. Phys. Chem. Chem. Phys. 2008, 10, 6615-6620. 